$\mathrm{O}-\mathrm{\square}$

\title{
Novel approaches for analyzing nanoparticles using Atom Probe Tomography
}

\author{
Pyuck-Pa Choi ${ }^{1, *}$ Se Ho Kim ${ }^{1,2}$, Kyuseon Jang ${ }^{1}$ \\ ${ }^{1}$ Department of Materials Science and Engineering, Korea Advanced Institute of Science and Technology, Daejeon, Korea \\ ${ }^{2}$ Department of Microstructure Physics and Alloy Design, Max-Planck-Institut fuer Eisenforschung, Duesseldorf, Germany \\ *corresponding author's e-mail: p.choi@kaist.ac.kr
}

(Received: January 11, 2019; Accepted: June14, 2019)

In this work, we present a novel approach for preparing atom probe tomography (APT) specimens from Pd electrocatalyst nanoparticles of less than $10 \mathrm{~nm}$ in size. This method is based on electrophoresis of nanoparticles on a substrate followed by electroplating of a metallic layer. Using transmission electron microscopy (TEM) we could observe that particle shape and size were well preserved after these two process steps. We could routinely prepare APT specimens from the deposited nanoparticle/metal films using focused-ionbeam milling (FIB). Using APT we were not only able to map the elemental distribution within the nanoparticles but also the distribution of surfactants i. e. stabilizing and shape-controlling agents, used in the synthesis of the nanoparticles.

\section{Introduction}

Atom probe tomography (APT) is a powerful tool for three dimensional chemical mapping of solids with near-atomic spatial resolution. It is based on the controlled field evaporation of surface atoms from a needle-shaped emitter and is a combination of ion projection microscopy and time-of-flight mass spectrometry. Impact positions and time-of-flight values of field-evaporated ions are recorded by a position-sensitive detector for reconstruction of a $3 \mathrm{~d}$ atom map, using an inverse projection algorithm. To date, most APT analyses have been performed on bulk or thin-film materials and only a few methods have been proposed for the investigation of nanoparticles by means of APT [1-3]. The fabrication of APT samples from nanoparticles poses a great challenge, where two main issues must be overcome. First, nanoparticles must be well embedded in a matrix suitable for APT analyses without any nano- or micro-voids. The latter can cause premature fracture during APT analyses and artifacts in APT data reconstruction and should be avoided. Second, structure and composition of the nanoparticles should be preserved as well as possible during the embedding process to acquire meaningful data, which can be related to the original freestanding nanoparticles. Here, we present a novel approach for preparing APT specimens from $\mathrm{Pd}$ electrocatalyst nanoparticles of less than $10 \mathrm{~nm}$ in size.

\section{Materials and Methods}

In order to prepare APT specimens, we first encapsulated the nanoparticles in an electrodeposited $\mathrm{Ni}$ film using combined electrophoresis and electrodeposition (schematically illustrated in Fig. 1). The Pd nanoparticles were found to be well embedded within the electrodeposited Ni film, as confirmed by scanning TEM (STEM) - energy dispersive X-ray spectroscopy (EDX) analyses (see Fig. 2).
The resulting Ni/Pd composite films could be further processed using FIB. A wedge-shaped bar of the film was prepared by FIB milling using rectangular milling patterns at a tilt angle of $22^{\circ}$. This bar was lifted out using a micromanipulator and placed on W support tips. Subsequently, needle-shaped APT specimens of less than $100 \mathrm{~nm}$ in diameter could be prepared using annular FIB milling. Prior to APT analyses, we examined the specimens using TEM in order to ensure that $\mathrm{Pd}$ nanoparticles were indeed included in the probed volumes.
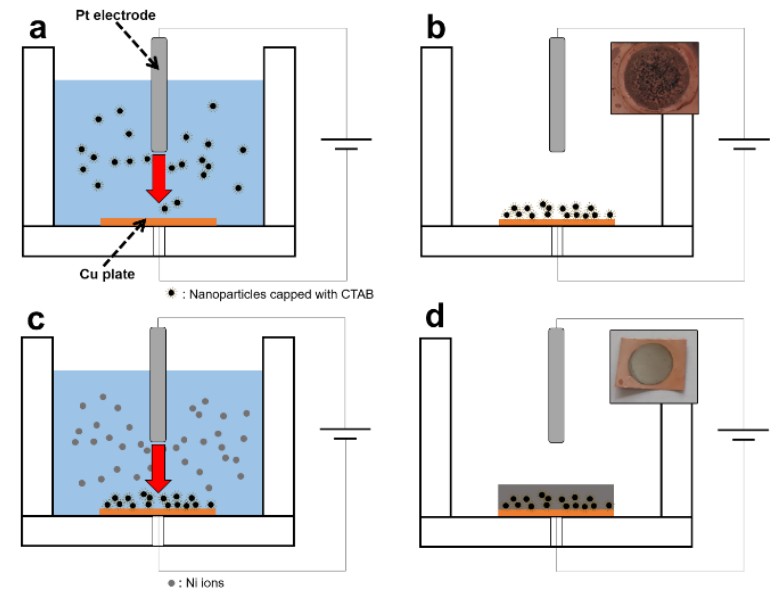

Fig. 1 Encapsulation of Pd nanoparticles in a Ni film, using combined electrophoresis and electrodeposition. 

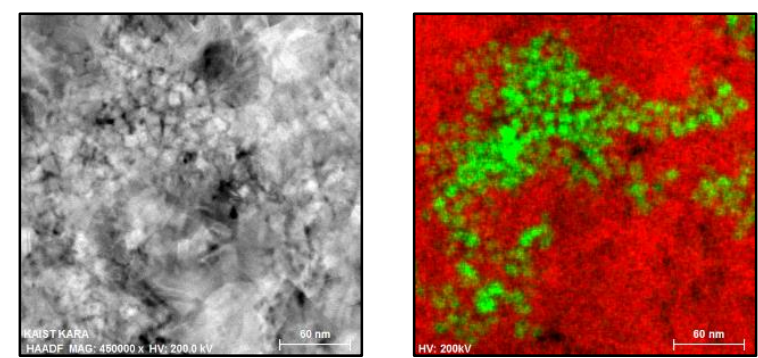

Fig. 2 STEM image (left) and STEM-EDX map (right) of Pd nanoparticles (green) embedded in the electrodeposited $\mathrm{Ni}$ film (red).

\section{APT results}

Fig. 3 shows a 3D atom map acquired from an APT analysis. Cuboidal Pd nanoparticles (yellow) are detected within the Ni matrix (green), demonstrating the feasibility of our specimen preparation approach. Interestingly, the nanoparticles exhibit enrichments of carbon-rich molecules at their corners and edges, as detected in the mass spectra. These complex molecules (marked in purple and light-blue in Fig. 3), are assumed to stem from organic additives, used in the nanoparticle synthesis. However, at this point we cannot unambiguously conclude to which organic additives they belong. Segregation of such organic compounds is most likely driven by a reduction in surface energy, which is expected to be highest at corner and regions. We expect our work to contribute to an improved understanding of the structure-composition-property relationships of nanoparticles.

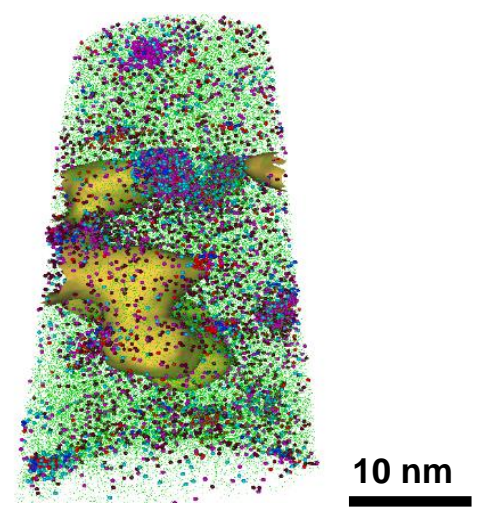

Fig. 3 APT dataset of Pd nanoparticles (highlighted in yellow) embedded in a Ni matrix (green). Organic molecules (purple and light blue) are detected as well.

\section{References}

[1] K. Tedsree, T. Li S. Jones, C. W. A. Chan, K. M. K. Yu, P. A. J. Bagot, E. A. Marquis, G. D. W. Smith, S. C. E. Tsang, Nat. Nanotechnol. 6, 302 (2011).

[2] P. Felfer, T. Li, K. Eder, H. Galinski, A. P. Magyar, D. C. Bell, G. D. W. Smith, N. Kruse, S. P. Ringer,
J. M. Cairney, U1tramicroscopy 159, 413 (2015).

[3] D. E. Perea, I. Arslan, J. Liu, Z. Ristanovic, L. Kovarik, B. W. Arey, J. A. Lercher, S. R. Bare, B. M. Weckhuysen, Nat. Commun. 6, 7589 (2015). 